\title{
MANUFACTURING OF PROTO TYPE WIND TURBINE BLADES USING
}

\section{RAPID PROTOTYPE TECHNOLOGY}

\author{
S. SELVAN NAMBI ${ }^{1} \&$ G. M. JOSELIN HERBERT ${ }^{2}$ \\ ${ }^{1}$ Research Scholar, Department of Mechanical Engineering, Noorul Islam Centre for Higher Education, \\ Thuckalay, Kanyakumari, Tamilnadu, India \\ ${ }^{2}$ Professor, Department of Mechanical Engineering, Noorul Islam Centre for Higher Education,
}

Thuckalay, Kanyakumari district, Tamilnadu, India

\begin{abstract}
All over the world, the wind energy has numerous benefits in helping to provide a source of clean and renewable electricity. For greater use of wind energy, the research efforts are aimed at addressing the challenge. The main objective of the project is to develop wind turbine blade model using CAD tool Autodesk inventor and Flash forge finder $3 D$ printer for without twist and with various twist angles of 30,60,90,120 and 150.2D and 3D data can be easily integrated with Autodesk inventor software in a single digital model to create a virtual representation of the final product. The wind turbine blades are fabricated through $3 D$ printing technology otherwise known as additive manufacturing process. The aim of this paper is to give an overview of this relatively infant technology, its diverse applications such as wind turbine industries for manufacturing prototype model to explain the development of new blade design.

KEYWORDS: Wind Turbine Blade, Rapid Prototyping Technology, Model, Materials \& Manufacturing
\end{abstract}

Received: Jan 02, 2018; Accepted: Jan 23, 2018; Published: Feb 26, 2018; Paper Id.: IJMPERDAPR201811

\section{INTRODUCTION}

In recent years, rapid prototyping technology (RPT) has been implemented in many spheres of industry, particularly in the area of product development. Existing processes provide the capability to rapidly produce a tangible solid part, directly from three dimensional computer aided design (CAD) data, from a range of materials such as photo curable resin, powders and paper. Many countries are working hard to get over the high-tech industries to upgrade engineering and manufacturing. There are very few technologies that offer as much as RPT. It is a new technology that transforms the digital designs into 3D solid objects.

\section{MATERIALS OF WTBS}

The selection of suitable materials for wind turbine blades (WTBs) is the quest for greater power and also to reduce the weight and increase the fatigue life. The important points and mechanical properties of WTBs materials are described as follows:

Lekon has described the topic of WTB materials using the probabilistic analysis. It has highlighted the issues arising out of the use of composite material (CM) for wind energy applications [1]. Clansen et al. have discussed the suitable materials for the manufacture of small WTBs (SWTBs) and large WTB (LWTB). The rotational speeds of WTBs have caused fatigue, stress and yaw moments. The timber materials and fiber reinforced composite materials (CMs) have been used for the SWTB and LWTB respectively for the better shape 
fatigue behavior [2]. Nijssen and Brondsted have addressed the suitability of CM for WTB manufacture. The pressure side and suction side of CMs have been subjected to tensile loads and compression loads respectively [3]. Madsen et al. have calculated the mechanical properties of bio-based composites cellulose fibers matrix materials using micromechanical model. The bio-based CMs have been considered for the manufacture of WTBs [4]. Faguaga et al. have studied the influence of water on the mechanical properties of Fiber Glass (FG) composite WTBs (CWTBs). The matrix degradation and interfacial de-bonding have been investigated using a dynamic mechanical technique. The presence of nano-clays have produced weak interface with the matrix. The FG / unsaturated polyester CWTBs have been produced by vacuum infusion technique (VIT) [5]. Wang et al. have analyzed the cross-sectional properties of modern CWTBs using a novel mathematical model. It has improved the accuracy of torsional stiffness owing to the shear web effects and warping effects of WTBs [6].

Hoevel and Sagnard have described the major issues pertaining to the use of new materials for the WTB manufacturing. The foam core material and epoxy resin materials have been used to tackle weight, strength and cure time of WTBs [7]. Marsh has overviewed the materials of LWTBs. The reinforced plastic has been used for the production of LWTBs in UK [8]. Ma and Yi have presented the perspectives of carbon nano tubes materials (CNTMs) owing to the higher strength to mass ratio. The mechanical, fatigue, electrical, thermal and barrier properties of CNTMs have been found more suitable for WTBs. It has been suggested for the commercialization of the CNTMs [9]. Rashedia et al. have studied the material selection aspects for on-shore and off-shore horizontal axis WTB and tower. They require design parameters have been obtained by the proper material selection [10].

\section{MANUFACTURING OF WTBS}

The processing technology of CMs must be developed for a boom of wind energy (WE). The technology, process and challenges have been considered for the manufacture of WTBs. They are briefly expressed as follows: Adolphs and Skinner have described the manufacture of WTB using the non-crimp fabric composites. It has explained the influences of fabric processing method [11]. Hollaway has introduced the properties and manufacturing techniques of WTBs made of fiber reinforced plastic (FRP). He has also suggested the repair, maintenance and recycling process of CWTBs [12]. Marsh has reported the advanced researches going on for the manufacture of WTBs made of thermoplastic. The report has explained the advantages of thermoplastic composite technology over the thermoset composite technology [13]. Langemeier and Scheuer have described the importance of resin for WTB manufacturing process. It has dealt with the big challenges and history of WTB manufacturing [14]. Subrahmanian and Dubouloz have discussed about the adhesives used for bonding of two shells together. The study has explained the major requirements, developments and properties of adhesives [15]. Larsen has presented the possible ways for the recycle of waste CMs (WCMs). The land fill, incineration and recycling have been used for waste disposals. $30 \%$ of the FRP waste could be reused to make a new FRP by recycling process. It is a boom for the WE development [16].

Ortegon et al. have discussed the end of service life of wind turbine generators (ESLWTGs) for the continuous development of WTG Technologies. The following prominent factors have been considered for the ESLWTGs. They are such as the fastest growing rate of the wind industry, remanufacturing, recycling, critical activities of reverse supply chain, economic and business motivation. The span of life of WTGs has been varied from 20 to 40 years [17]. Papadakis et al. have examined the scenarios of the ESLWTG. The management of WCMs has involved the safe disposal and recycling process [18]. Schubel has conducted a detailed technical cost modeling for a generic 45m WTB produced by VIT. 
The material and labour costs have occurred nearly $51 \%$ and $41 \%$ respectively. The blade cost has been saved by the fluctuations in reinforcement, improving material deposition time and cure time [19]. Schubel has performed a cost modeling and analyzed the manufacturing processes of a LWTB. The automated blade production methods have reduced the manufacturing costs by up-to $8 \%$ and it has also reduced the number of labor requirement [20]. Hutchinson et al. have done a cost and performance comparisons between the light resin transfer molding (LRTM) and VIT of WTBs. The LRTM technique has more advantages about $4 \%$ increase in mechanical performance, $3 \%$ cost saving, $5.5 \%$ improvement in dimensional stability, $3 \%$ reductions in resin wastage and $25 \%$ infusion time [21]. The new manufacturing concepts have been adapted to enable the viability of LWTBs. The aerodynamic design parameters have been importantly considered for the manufacture of efficient WTBs. The proper and timely maintenance practices have to be carried out followed by manufacturing and operation of WTBs.

\section{TECHNOLOGY OVERVIEW}

A group of technologies which are capable of performing the process almost under computer control, with the help of human or maybe not has begun. The RPT process can be classified into five criteria such as

- In this process shapeless form raw material such as sheets, blocks or a fluid should be converted into a definite shape solid object.

- $\quad$ There should be less amount of human interaction during the process.

- With some degree of three dimensional geometrical complexities the process must produce shapes. This helps to eliminate the forming of tubes or rods by extrusion and cutting or drilling holes in sheet material.

- The manufacturing of new tools should not be involved for different shapes which are to be generated.

- The item which is to be produced should be a single object and not an assembly of component.

Well there are not techniques satisfying the automated formative criteria because, if we want to form a shape, a unique combination of mechanical forces is too applied to the raw material. The fact that rapid prototyping processes are based on automated additive fabrication means they represent a total departure from conventional manufacturing techniques. Such a departure obviously yields a unique set of problems to be addressed.

\section{TERMINOLOGY}

The RPT is still an infant technology because it has yet to be adopted.

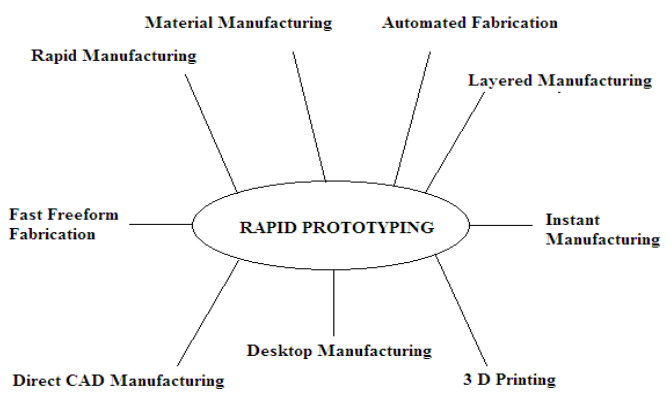

Figure 1: Terminology Associated with Rapid Prototyping 


\section{LAYERED MANUFACTURING: PROCESS FUNDAMENTALS}

Based on a layered manufacturing methodology in which objects are built as a series of horizontal cross sections. Each one being formed individually from the relevant raw materials and bonded to preceding layers until it is completed. The processes of fabricating parts are common to most systems, but the mechanisms which are created depend on the particular system. This data must be manipulated to generate the instructions required to control the process in the final stage of actually fabricating the component.

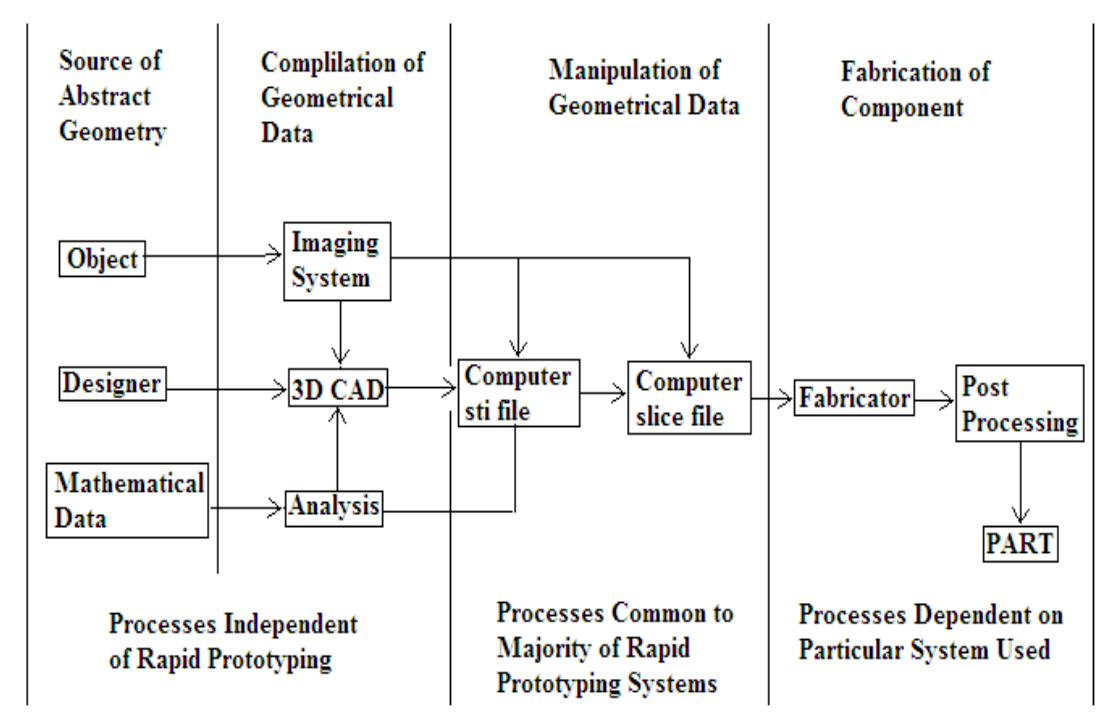

Figure 2: Main Process Stages Common to Most Rapid Prototyping Systems

\subsection{Issues in Layered Manufacturing}

The method of manufacturing objects as a series of horizontal layers poses a unique set of problems, irrespective of the techniques involved in the fabrication of each layer. Some of these problems are technical and may be as a result of hardware limitations while other may be due to the finishing operations.

\subsubsection{The "Stair-Stepping" Phenomenon}

A set of horizontal cross section is produced by the slicing process and in horizontal plane, these sections conforms to geometry of the original CAD model. The parts cannot accurately conform to the CAD geometry in the vertical plane because each layer is in continuous cross section through its thickness.

\subsubsection{Layer Thickness Selection}

The smaller slice thickness, the greater the resolution of the final part. It may seem sensible to use the smallest possible layer thickness that can be physically created by the system, but the smaller the layer thickness, the more slices are required resulting in longer data processing time, larger data files and a longer build time. To optimize the process, variable layer thickness may be used over different ranges of the part.

\subsection{Rapid Manufacturing Technology}

It is a manufacturing technology that refers to built by rapid prototyping systems. It can be used to fabricate metallic components. 


\subsubsection{Rapid Tooling Technology}

It is an important application for the production of prototype tooling.

\subsection{Benefits of Rapid Prototyping Technology}

There is reduction in errors on the model through improved part visualization. There is an average savings of $80 \%$ both in cost and time over the traditional methods. In addition, a significant improvement in the acoustic / mechanical properties was noted in comparison to vacuum casting parts. Another important benefit of RP\&M technology is the facilitation of concurrent engineering, which has become a powerful and useful tool in design and product development in industries.

\subsection{D Printing Technology}

It is also known as additive manufacturing (AM). It is a process which is used for the creation of 3D object in which the layers are formed under the control of a computer for creating an object. The objects which are made can be of any geometrical shape and are mostly produced by using digital model data.

\subsection{Principles of 3D Printing Technology}

\subsubsection{Modelling}

With the help of a CAD package, photogrammetric software, by a 3D scanner, a 3D printable model can be created. There will be fewer errors if the 3D printed models are created with CAD. 3D scanner can be used for collecting digital data on the shape and creating a digital model based on it.

\subsubsection{Printing}

For carrying out printing a 3D model from an STL file the error should be examined first. Errors are produced in output STL files in most of the CAD applications includes holes, faces normal, self-intersection, noise shells and manifold errors.

\subsubsection{Finishing}

The resolution produced for the printer is sufficient for most of the applications. There are some printable polymers which allow the surface finish to be smoothed and more improved by using chemical vapour processes.

In the course of constructing part some of the additive manufacturing techniques having the capability to use multiple materials. It does not necessarily require painting, because this techniques having the capability to print multiple colors and color combination simultaneously. For building overhanging features during construction require internal support to be built in some printing techniques. After completion of the print these supports must be mechanically removed or dissolved. Cutting the metal component of the metal substrate after deposition is involved in all of the commercialized metal 3D printers. To remove aluminium or steel a new process GMAW printing allows for substrate surface modifications.

\subsubsection{Applications}

3D printing or AM has been used in medical, manufacturing and social cultural sectors in the current scenario. It becomes successful commercial technology. AM of food is being developed by squeezing of food layer by layer into $3 \mathrm{D}$ objects such as can be candy, chocolate and flat food such as pasta, crackers and pizza. It also entered in the world of 
clothing with fashion designers. They are experimenting with 3D printed shoes, dresses and bikinis. For bony reconstructive surgical planning uses 3D printing centric therapies with anatomical modeling. Hobbyists and enthusiasts practicing domestic 3D printing for practical household applications such as ornamental objects, working clock and gears printed for home woodworking machines etc. Future applications for 3D printing might include creating open-source scientific equipment. This technology has been used in the cultural heritage field for the last several years for the purpose of restoration, preservation and dissemination.

\subsection{Flash Forge Finder}

3D printing with the finder is more affordable and easier. It has the capability of printing right of the box with a few tweaks. The printed material used is non-toxic polylactic acid (PLA) and all the heated elements are protected from touch.

During manufacturing the noise level is as low as $50 \mathrm{~dB}$ or even less. Second generation Wi-Fi connection with the more stable file transmission is one of the important feature of finder. Now we can send 3D files from our computer to finder with 4GB memory with our own Wi-fi network under STA or AP modes.

Another stable way to transfer file is by using USB stick. When the computer runs into a stand by no USB cable or no interruption should be made. On the finder the filament management is rather impressive. The flash forge PLA filament is able to detect when the filament is running out by a cartridge housing of $1.75 \mathrm{~mm}$. It alerts to load new filament instead of getting stuck with extruding nothing. If we turnoff in the function in the settings the finder can be used with third party filament.

\subsection{Software Used}

The Software used for 3D printing is flash print. For converting one design to a 3D printed modeled a simple and intuitive interfaces are used for beginners. It has a list of preset for built quality. We have to select a built quality what we want and flash print take care of the rest. It has more control over the software for changing its parameter, temperature, printing speed, first layer thickness and height when the printing is pursing by manually. We can get layer by layer visualization, time and material estimate by slicing. So that generation becomes easier than that of any other techniques. More complex models can be print by Dentri form or treelike supports which can be snapping of supports easily without damaging the object. When the object is too big as for one print, it should be divide the model into several separate parts. Flash print featured with cut and split function for this. The bigger models can be print by the limited built volume. For converting 2D image file into 3D models the 2D into 3D featured in the flash print used for this. This is the easiest way to start 3D designing. For this what we need is to drag the image and select parameters you want.

\section{BLADE DESIGN}

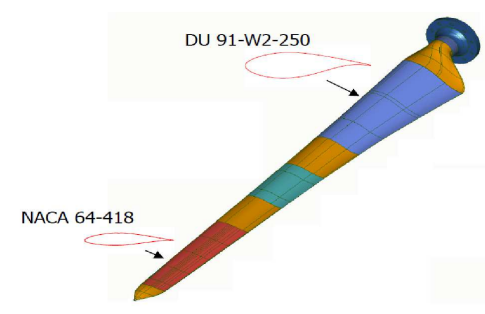

Figure 3: Modified LM Blade 
The blade structure is generated using CATIA software for without twist and with a twist of $(30,60,90,120,150)$ angles.

\section{BLADE MANUFACTURE IN RAPID PROTO TYPE 3D PRINTING}

The designed blade structure using CATIA software was converted into STL format and fed as an input to the 3D flash printer through USB port. The rolled wire PLA material is fed into the machine from the roller.

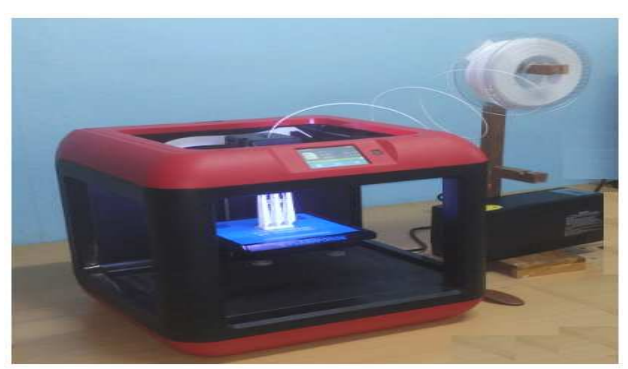

Figure 4: Rapid Proto Type 3D Printing

The wire is heated in the temperature range of 220 to $2200 \mathrm{C}$, at which it melts and the designed WTB shape is formed based on the fed shape. The proto type model was manufactured by depositing melted PLA thermo plastic material layer by layer by using Rapid proto type 3D printing.

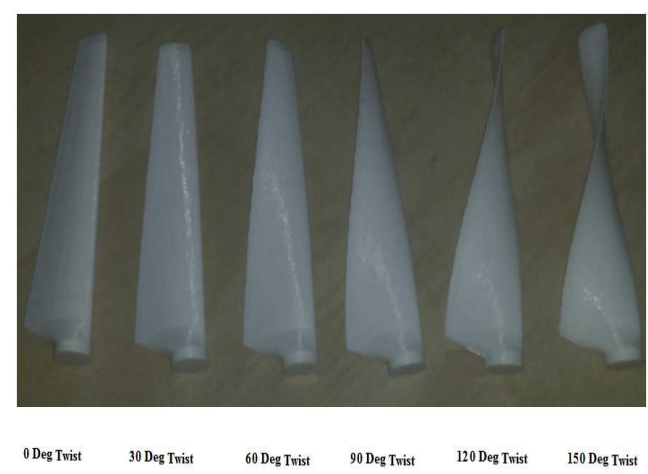

Figure 5: Wind Turbine Blades with Various Twist Angles

The wind turbine blades models without twist and with a twist of 30, 60, 90120 and 150 degree were manufactured in RPT - 3D printing

\section{CONCLUSIONS}

The renewable and sustainable wind energy is completely free and it makes it viable option for generating cheap electricity and running costs are considered to be low. The production cost for manufacturing actual WTB is very high. For demonstrating the new modeled WTB it is better to produce prototype model for the scaled dimensions for the actual one. By the $3 \mathrm{D}$ printing the following are the advantages.

- It will save material.

- $\quad$ Production time and cost decreases.

- The design and manufacturing fault can be easily identified. 
- The required alteration can be made before the actual blade is produced.

- $\quad$ Able to produce parts and concept models using desktop printer.

- $\quad$ More complex objects can be produced cheaply.

However, in the modern methods the construction of a model can take several hours to several days depending on the method, size and complexity of the model used.

\section{REFERENCES}

1. Lekou DJ. Advances in WTB design and Materials, A volume in WHPS in Energy. Chapter 10 -Probabilistic design of WTBs. 2013, 325-59. Edited by Brondsted. P, Nijessen R. ISBN: 978-0-85709-426-1.

2. Clausen PD, Reynal F. Wood. D. H. Advances in WTB Design and Materials, A volume in WHPS in Energy. Chapter 13 -Design, manufacture and testing of small WTBs. 2013; 413-31. Edited by Brondsted. P, Nijessen R. ISBN: 978-0-85709-426-1.

3. Nijssen RPL. Brondsted. P. Advances in WTB Design and Materials. A volume in WHPS in Energy. Chapter 6 -Fatigue as a design driver for CWTBs. 2013, 175-209. Edited by Brondsted. P, Nijessen R. ISBN: 978-0-85709-426-1.

4. Madsen B, Brondsted P, Logstrup Andersen T. Advances in WTB Design and Materials, A volume in WHPS in Energy. Chapter 11 -Biobased composites: materials, properties and potential applications as WTB materials. 2013; 363-86. Edited by Brondsted. P, Nijessen R. ISBN: 978-0-85709-426-1.

5. Faguaga E, Perez CJ, Villarreal N, Rodriguez ES, Alvarez V. Effect of water absorption on the dynamic mechanical properties of composites used for windmill blades. Materials \& Design 2012; 36(4):609-16.

6. Wang L, Liu X, Guo L, Renevier N, Stables M. A mathematical model for calculating cross-sectional properties of modern CWTBs. Renewable Energy 2014; 64(4):52-60.

7. Hoevel B, Sagnard A. New materials tackle weight, strength and cure time issues for WTBs. Reinforced Plastics 2011; 55(4):38-41.

8. $\quad$ Marsh G. Tooling up for large WTBs. Reinforced Plastics 2007; 51(9):38-40, 42-43.

9. Ma PC, Zhang Y. Perspectives of carbon nanotubes/polymer nanocomposites for wind blade materials. Renewable and Sustainable Energy Reviews 2014; 30(2):651-60.

10. Ashwani Sharma \& M. A. Murtaza, Modeling and Finite Element Analysis of Vertical Axis Wind Turbine Rotor Configurations, International Journal of Mechanical and Production Engineering Research and Development (IJMPERD), Volume 6, Issue 3, May-June 2016, pp. 23-34

11. Rashedi A, Sridhar I, Tseng KJ. Multi-objective material selection for WTB and tower: Ashby's approach. Materials \& Design 2012; 37(5):521-32.

12. Adolphs $G$, Skinner C., Manufacturing, properties and applications, A volume in WHPS in Composites Science and Engineering. Chapter 21 -Non-crimp fabric composites in wind turbines, 2011; 48-493. Edited by Lomov SV. ISBN: 9781845697624. Available online 29 Jan 2014.

13. Hollaway LC. A volume in WHPS in Civil and Structural Engineering. Chapter 20-Advanced fibre-reinforced polymer (FRP) CMs for sustainable energy technologies. 2013; 737-79. Ediited by Bai J. ISBN: 978-0-85709-418-6. Available online 31 Jan 2014.

14. Marsh G. Could thermo plastics be the answer for utility-scale WTBs. Reinforced Plastics 2010; 54(1):31-35. 
15. Langemeier P, Scheuer C. Big challenges: the role of resin in WTB development. Reinforced Plastics 2010; 54(1):36-39.

16. Subrahmanian KP, Dubouloz F. Adhesives for bonding WTBs. Reinforced Plastics 2009; 53(1):26-9.

17. Larsen K. Recycling WTBs. Renewable Energy Focus 2009; 9(7):70-73.

18. Ortegon K, Nies LF, Sutherland JW. Preparing for end of service life of wind turbines. J. of Cleaner Production 2013; 39(1):191-99.

19. Papadakis $N$, Ramirez C, Reynolds N. Management. Recycling and reuse of waste composites, A volume in WHPS in Composites Science and Engineering. Chapter 16-Designing CWTBs for disposal, recycling or reuse 2010; 443-57. Edited by Goodship V. ISBN: 978-1-84569-462-3.

20. Schubel PJ. Technical cost odeling for a generic 45m WTB produced by vacuum infusion (VI). Renewable Energy 2010; 35(1):183-89.

21. Schubel PJ. Cost odeling in polymer composite applications: Case study-Analysis of existing and automated manufacturing processes for a large WTB, Composites Part B: Engineering 2012; 43(3):953-60.

22. Hutchinson JR, Schubel PJ, Warrior NA. A cost and performance comparison of LRTM and VI for the manufacture of large scale WTBs. Renewable Energy 2011; 36(2):866-71.

23. Selvan Nambi S. Joselin Herbert GM. Analysis of new modeled twisted WTBs. International Journal of Mechanical Engineering and Technology. 2017; 8(12):1148-66. 
